\title{
Correction to: The Japanese Breast Cancer Society Clinical Practice Guidelines for systemic treatment of breast cancer, 2018 edition
}

\author{
Tatsunori Shimoi ${ }^{1,23} \cdot$ Shigenori E. Nagai ${ }^{2,23} \cdot$ Tetsuhiro Yoshinami $^{3,23} \cdot$ Masato Takahashi $^{4,23} \cdot$ Hitoshi Arioka $^{5,23}$. \\ Mikiya Ishihara ${ }^{6,23} \cdot$ Yuichiro Kikawa $^{7,23} \cdot$ Kei Koizumi $^{8,23} \cdot$ Naoto Kondo $^{9,23} \cdot$ Yasuaki Sagara $^{10,23}$. \\ Masahiro Takada ${ }^{11,23} \cdot$ Toshimi Takano $^{12,23} \cdot$ Junji Tsurutani ${ }^{13,23} \cdot$ Yoichi Naito $^{14,23} \cdot$ Rikiya Nakamura $^{15,23}$. \\ Masaya Hattori ${ }^{16,23}$. Fimikata Hara ${ }^{17,23}$. Naoki Hayashi ${ }^{18,23}$. Toshiro Mizuno ${ }^{6,23}$. Minoru Miyashita ${ }^{19,23}$. \\ Nami Yamashita ${ }^{20,23} \cdot$ Takashi Yamanaka $^{21,23} \cdot$ Shigehira Saji ${ }^{22,23} \cdot$ Hiroji Iwata $^{16,23} \cdot$ Tatsuya Toyama $^{9,23}$
}

Published online: 22 April 2021

(c) The Author(s) 2021

\author{
Correction to: Breast Cancer (2020) 27:322-331 \\ https://doi.org/10.1007/s12282-020-01085-0
}

The original article can be found online at https://doi.org/10.1007/ s12282-020-01085-0

Tatsuya Toyama

t.toyama@med.nagoya-cu.ac.jp

1 Department of Breast and Medical Oncology, National Cancer Center Hospital, 5-1-1 Tsukiji,, Chuo-ku, Tokyo 104-0045, Japan

2 Department of Breast Oncology, Saitama Cancer Center, 780 Komuro, Ina-machi, Kitaadachi-gun, Saitama 362-0806, Japan

3 Department of Breast and Endocrine Surgery, Graduate School of Medicine, Osaka University, 2-2-E 10 Yamadaoka, Suita, Osaka 565-0871, Japan

4 Department of Breast Surgery, NHO Hokkaido Cancer Center, 4-2 Kikusui, Shiroishi-ku, Sapporo 003-0804, Japan

5 Department of Medical Oncology, Yokohama Rosai Hospital, 3211 Kozukue, Kohoku-ku, Yokohama, Kanagawa 222-0036, Japan

6 Department of Medical Oncology, Mie University Hospital, 2-174 Edobashi, Tsu, Mie 514-8507, Japan

7 Department of Breast Surgery, Kobe City Medical Center General Hospital, 2-1-1, Minatojimaminamimachi, Chuo-ku, Kobe, Hyogo 650-0047, Japan

8 First Department of Surgery, Hamamatsu University School of Medicine, 1-20-1 Handayama, Higashi-ku, Hamamatsu City, Shizuoka 431-3192, Japan

9 Department of Breast Surgery, Nagoya City University Graduate School of Medical Sciences, 1 Kawasumi, Mizuho-cho, Mizuho-ku, Nagoya 467-8601, Japan

10 Department of Breast Surgical Oncology, Hakuaikai Social Cooperation, Sagara Hospital, 3-31 Matsubara-cho, Kagoshima 892-0098, Japan
11 Department of Breast Surgery, Kyoto University Hospital, 54 Kawaharacho, Shogoin, Sakyo-ku, Kyoto 606-8507, Japan

12 Department of Medical Oncology, Toranomon Hospital, 2-2-2 Toranomon, Minato-ku, Tokyo 105-8470, Japan

13 Department of Medical Oncology, Advanced Cancer Translational Research Institute, Showa University, 1-5-8 Hatanodai, Shinagawa, Tokyo, Japan

14 Department of Breast and Medical Oncology, National Cancer Center Hospital East, 6-5-1 Kashiwanoha, Kashiwa, Chiba 277-8577, Japan

15 Department of Breast Surgery, Chiba Cancer Center, 666-2 Nitona-cho, Chuo-ku, Chiba, Chiba 280-8717, Japan

16 Department of Breast Oncology, Aichi Cancer Center, 1-1 Kanokoden, Chikusa-ku, Nagoya 464-8681, Japan

17 Department of Breast Medical Oncology, The Cancer Institute Hospital of the Japanese Foundation for Cancer Research, 3-8-31 Ariake, Koto-ku, Tokyo 135-8550, Japan

18 Department of Breast Surgical Oncology, St. Luke's International Hospital, 9-1 Akashi-cho, Chuo-ku, Tokyo 104-8560, Japan

19 Department of Breast and Endocrine Surgical Oncology, Tohoku University Graduate School of Medicine, Sendai, Miyagi 980-8575, Japan

20 Department of Surgery and Science, Kyushu University, 3-1-1 Maidashi, Higashi-ku, Fukuoka 812-8582, Japan

21 Department of Breast and Endocrine Surgery, Kanagawa Cancer Center, 2-3-2 Nakao, Ashahi-ku, Yokohama 241-8515, Japan

22 Department of Medical Oncology, Fukushima Medical University, 1 Hikarigaoka, Fukushima 960-1295, Japan

23 The Japanese Breast Cancer Society Clinical Practice Guidelines for Systemic Treatment of Breast Cancer Panel Membership, Tokyo, Japan 
The article "The Japanese Breast Cancer Society Clinical Practice Guidelines for systemic treatment of breast cancer, 2018 edition", written by Tatsunori Shimoi, Shigenori E. Nagai, Tetsuhiro Yoshinami, Masato Takahashi, Hitoshi Arioka, Mikiya Ishihara, Yuichiro Kikawa, Kei Koizumi, Naoto Kondo, Yasuaki Sagara, Masahiro Takada, Toshimi Takano, Junji Tsurutani, Yoichi Naito, Rikiya Nakamura, Masaya Hattori, Fimikata Hara, Naoki Hayashi, Toshiro Mizuno, Minoru Miyashita, Nami Yamashita, Takashi Yamanaka, Shigehira Saji, Hiroji Iwata, Tatsuya Toyama, was originally published electronically on the publisher's internet portal on 2 April 2020 without open access. After publication in volume 27 , issue 3 , page 322-331, the author decided to opt for Open Choice and to make the article an Open Access publication. Therefore, the copyright of the article has been changed to (C) The Author(s) and the article is forthwith distributed under the terms of the Creative Commons Attribution 4.0 International License, which permits use, sharing, adaptation, distribution and reproduction in any medium or format, as long as you give appropriate credit to the original author(s) and the source, provide a link to the Creative Commons licence, and indicate if changes were made.

The images or other third party material in this article are included in the article's Creative Commons licence, unless indicated otherwise in a credit line to the material. If material is not included in the article's Creative Commons licence and your intended use is not permitted by statutory regulation or exceeds the permitted use, you will need to obtain permission directly from the copyright holder.

To view a copy of this licence, visit http://creativeco mmons.org/licenses/by/4.0/.

The original article has been updated.

Open Access This article is licensed under a Creative Commons Attribution 4.0 International License, which permits use, sharing, adaptation, distribution and reproduction in any medium or format, as long as you give appropriate credit to the original author(s) and the source, provide a link to the Creative Commons licence, and indicate if changes were made. The images or other third party material in this article are included in the article's Creative Commons licence, unless indicated otherwise in a credit line to the material. If material is not included in the article's Creative Commons licence and your intended use is not permitted by statutory regulation or exceeds the permitted use, you will need to obtain permission directly from the copyright holder. To view a copy of this licence, visit http://creativecommons.org/licenses/by/4.0/.

Publisher's Note Springer Nature remains neutral with regard to jurisdictional claims in published maps and institutional affiliations. 